\title{
Demographic data for development in sub-Saharan Africa
}

\author{
Wendy Baldwin \\ Population Council \\ Judith A. Diers \\ Population Council
}

Follow this and additional works at: https://knowledgecommons.popcouncil.org/departments_sbsr-pgy

Part of the Demography, Population, and Ecology Commons, Family, Life Course, and Society Commons, and the International Public Health Commons How does access to this work benefit you? Let us know!

\section{Recommended Citation}

Baldwin, Wendy and Judith A. Diers. 2009. "Demographic data for development in sub-Saharan Africa," Poverty, Gender, and Youth Working Paper no. 13. New York: Population Council. 


\section{POVERTY, GENDER, AND YOUTH}

\section{Demographic Data for Development in Sub-Saharan Africa}

Wendy Baldwin and Judith Diers

WORKING PAPER NO. 132009

$\mathcal{Q}$ Population Council 


\section{(2) Population Council}

One Dag Hammarskjold Plaza

New York, New York 10017 USA

www.popcouncil.org

pubinfo@popcouncil.org

For information on Poverty, Gender, and Youth working papers, see www.popcouncil.org/publications/wp/index.html

This material may not be reproduced without written permission from the authors.

ISSN: $1554-8538$

(C) 2009 The Population Council, Inc. 


\title{
Demographic Data for Development in Sub-Saharan Africa
}

\author{
Wendy Baldwin \\ Judith Diers
}

Wendy Baldwin is Director and Judith Diers is Associate, Poverty, Gender, and Youth Program, Population Council, New York.

The research was supported by the William and Flora Hewlett Foundation. The views and opinions expressed in this paper are those of the authors and do not necessarily represent those of the Hewlett Foundation. 


\begin{abstract}
More demographic data are being collected throughout the developing world than ever before, but the effective use of that data to further development goals is often lacking. This paper summarizes case studies on the demand for data in four sub-Saharan African countries, namely Ethiopia, Ghana, Senegal, and Uganda. The project's objective was to create a detailed portrait of access and demand at the country level, and to determine whether policymakers are getting the data they need to develop sound policies. Common findings across the four countries include an increased external demand from international initiatives that has not necessarily translated into internal demand for data; a missing link between producers and users of data; and a need for data to be presented in user-friendly formats. One driver of internal demand for data is the decentralization and democratization process that is underway in all four countries; this demand highlighted the paucity of available data that can be disaggregated at the level to which policies were being devolved. Next steps are to support initiatives to establish data access as a right, encourage a culture of data-sharing among funders and producers of data, strengthen intermediaries between policymakers and data collectors, display data in accessible formats such as maps, and disaggregate available data to the most useful levels.
\end{abstract}


Demographic data provide an essential evidence base for the development and evaluation of public policies. The bottom line is that a country cannot develop without data. Although the term "data" has technical overtones and important technical issues need to be considered, the more fundamental connection is between good data and good governance. It is in part through the collection and analysis of data that countries and communities have defined themselves, identified inequities, challenged misconceptions, and justified their claims upon resources. Accessible data function as a public good, benefiting citizens and the political system as a whole. It is not by accident that the world's largest democracy-India-has adopted legislation giving citizens the right to demand access to information, and similar legislation is being developed in Ghana. Yet legislation alone ensures neither data-inspired policymakers nor a public empowered to hold them accountable.

The good news is that more data are being collected throughout the developing world than ever before; but how are these data being used? Is demand for data emerging from within countries, or is it largely being expressed by outsiders? Of course, the evidence base is only one of many factors that influence policy development, not all of which can be addressed in this working paper.

The Demographic Data for Development project ${ }^{1}$ examined the demand for data in four sub-Saharan African countries. The project focused on the views of policymakers, what data they use, what data they want but do not receive, and what data they might use but do not request. We further explored the underutilization of existing data and asked about the factors that limit the collection, use, and sharing of data. Our case studies sought to understand how access to data might be improved. The project's objective was to create a detailed portrait of access and demand at the country level, thereby complementing the international initiatives (such as PARIS $21^{2}$ and the Health Metrics Network ${ }^{3}$ ) that are underway. Although the emphasis was on demographic data, the project covered the range of data that are fundamental to developing and assessing policies in the areas of education, health, environment, income, employment, and other measures of development. Specifically, these case studies inquired about censuses, surveys, and both budget and service use statistics to determine whether policymakers are getting the data they need to develop sound policies.

Semi-structured interviews were conducted with over 100 policymakers, data producers, and data consumers in Ethiopia, Ghana, Senegal, and Uganda. This working paper presents the broad perspective from the four countries, with notes about where issues were raised specifically for one country or another. These countries were selected on the basis of their statistical capacity, upcoming censuses, active engagement in the production of data, and participation in international data initiatives. Illustrative quotations have been included, but identifying information has been omitted to preserve the anonymity of respondents. By reaching out to policymakers, government agencies, civil society organizations, other NGOs, development partners, researchers, and the media, these case studies developed a broad view of the relevant issues. 


\section{DETERMINANTS OF DEMAND}

Demand for data is perhaps the lynchpin of efforts to increase the quantity and quality of data. Assessing the level of demand for data is challenging given the different definitions of data and of demand. However, one thing is clear: demand for data is increasing. The case studies brought to light some significant determinants of demand: international influence, decentralization, and democratization.

\section{International influence}

Global initiatives to improve health and well-being in the developing world are increasingly quantified, relying on data to reveal trends and assess progress toward development goals. Recent initiatives such as the Poverty Reduction Strategy Papers are requiring major data collection efforts, which are effectively increasing demand. A government respondent emphasized that statistics have become particularly important in light of the need to monitor progress: "The pressure to report on the MDGs [Millennium Development Goals] in particular has influenced data priorities and heightened the focus on monitoring and evaluation."

The adoption of results-based budgeting by international donors has generally had a positive influence on demand; as one development partner said, "Emphasis on measuring impact is really pushing demand and calls for sophisticated data collection and analysis." In addition, international initiatives have created greater awareness of demographic indicators such as maternal mortality: "Some African countries would not have made maternal mortality a priority had it not been for the MDGs." Within the four countries studied, there is considerable reliance on the Demographic and Health Surveys (DHS) and high regard for the quality of these data and the protocols the DHS has established for making the data easily available. Moreover, it appeared that the DHS had generally contributed to the development of local capabilities. One interviewee mentioned that his staff gained skills in study design, data collection, and analysis through participation in the DHS.

The case studies caution us that while international demand for data has had some benefits, sustainable data

The international approach of linking investments with measurable outcomes often places great pressure on developing countries. These countries may be ill-equipped to produce the requisite data, and the consequences can be severe. Interviewees reported that their country recently lost 30 million Euros in funding because of an inability to produce data showing that the relevant targets had been met. systems require strong internal demand. As one researcher noted, "Much of the demand is donor driven. Indigenous demand remains weak. Up to now, the main reason for collecting data was to satisfy donor demand, rather than for our own planning needs." Externally driven and internationally funded surveys might, in the long term, divert resources from activities or topics of local or national interest and could potentially undermine the development of core national data systems, such as the census. In one government interview, we were told that: "In [an international survey], we could not include some locally relevant indicators/variables onto the 
questionnaire, as it allowed only little flexibility and also because it was already overstuffed to accommodate more questions. That was a missed opportunity." Interviewees in the four countries perceived internal demand as essential: "Indigenous demand is the only sustainable way to ensure better-quality data, access, and use." Since local investment in data is critical to the long-term health of the system, this national-international tension bears watching.

\section{Decentralization and democratization}

The international (external) demand for data exists alongside internal demand from the countries themselves. A move toward decentralization and democratization is underway in each of the countries studied and has catalyzed internal demands for data. Increasingly, governments are developing decentralized strategies for planning, budgeting, and budget execution. In the words of one parliamentarian, "Fundamentally, the statistical system should adapt itself to the requirements of the new development (decentralization) framework." In theory, decentralization shifts responsibilities to the municipal/district/local levels and reinforces the need for data. In

\begin{tabular}{|l|}
\hline Government \\
officials in Uganda \\
participate in short \\
courses on \\
statistics in order \\
to learn to use and \\
appreciate the \\
value of data. \\
\hline
\end{tabular}
reality, decentralization has proceeded by fits and starts, impeded by lack of capacity at the lower levels of government. Until these levels of government develop their own capacities, the motivation for national authorities to provide local-level data may be minimal.

Meanwhile, democratization ideally creates a demand for data as a means to hold government officials accountable. It has the potential to create pressure from below for better data, more timely data, and data that can influence both local and national decisions. Perhaps nowhere is the need for access to data so closely tied to the democratic process as with budget data. Respondents continually referred to the need for transparency in budgetary information. As one government official noted, "The central government has been publishing releases of fundsshowing amounts of funds released-but this is not enough. For example, if the funds are for roads, the information should indicate which roads - not just a lump sum for roads." This need for fine-grained budget data is an important means by which governments are held accountable, and respondents perceived that significant changes are underway in what people want to know about budget data: "People now demand detailed information compared to the situation before; people just used to report 'this is the budget,' but now people are asking 'to whom was it released and for what purpose?"”

\section{IMPEDIMENTS TO DEMAND}

Despite the number of opportunities to increase demand for demographic data collection and dissemination, several obstacles remain. An overriding issue-indeed, one that is seen in rich and poor countries alike - is that policies are often driven by political views rather than by empirical analysis. In rich countries, however, quantitative data provide a means by which such ill-founded policies can be challenged. Only the beginnings of this role for data are evident in the four study countries, where we found lack of data, lack of awareness of and access to existing 
data, poor data quality and lack of timeliness, inadequate resources, and missing links between data producers and users.

\section{Political influence}

In an ideal world, policy development would always take into account the evidence base. In reality, policymakers need to balance a wide range of factors and pressures. Data may be ignored if they challenge policymakers' personal views or political needs. In cases where policymakers do seek out data, this might be done after the fact to support policies already enacted. In one country we studied, a research organization reported seeing the greatest demand for data during election season; another respondent seconded this notion by saying, "It appears that data are quoted for the sake of elections rather than for planning." The consequences of not considering the evidence can be detrimental: "It is like crisis management. The policies are not well thought out, and that is why sometimes we get policies that do not stand the test of time." Until a culture develops whereby data are used to inform policy, the demand for data collection and use will remain low among policymakers themselves.

Even when national policies become evidence-based (at the stage when many interested parties and key stakeholders are involved and the available data are reviewed), problems can arise

For most of 2008, the Central Statistics Agency in Ethiopia was developing a National Strategy for Statistical Development to create a standardized framework for statistical reporting and increased statistical capacity. In October 2008, over 100 stakeholders met to assess the progress of the national strategy and began to implement its major recommendations. during policy implementation, when data are often ignored. As noted in one country: "Policies are made for the shelves. We hardly implement. You find that the two are not related at all. When it comes to the implementation of programs, there is very little or weak linkage with available data to inform those programs."

\section{Lack of awareness}

In some instances data are available, but users are unaware of their existence and/or their potential. One respondent asked, "How can people use data if they don't know that the data exist?" Respondents pointed to poor dissemination as one cause. Another respondent mentioned a common complaint that foreigners often have greater access to a country's data than citizens themselves. However, the core concern here is that unlike local researchers, foreign researchers come from bureaucratic and statistical cultures in which access to data is assumed. A respondent said, "Outsiders get data because they look for it; as a people we are not in the habit of finding and using data." Respondents repeatedly mentioned the lack of a reading culture in their countries as another constraint to data awareness. In the words of one respondent, "People abhor in-depth reading," and this cultural tendency deters them from actively seeking information. Data awareness is further constrained because the existence of data is often not widely known incountry; consequently, additional data are collected to fill a perceived void: "We usually rush to conduct surveys without exploring what data are available; we do not even know the institutions 
working in the area." Our case studies highlighted the fact that data are housed in multiple ministries and agencies, with no single organization acting as a clearinghouse.

The demand for data is also built on an awareness of their potential value, not just their existence. There is great potential in creating awareness of data and the possibilities for their application not only among policymakers, but also among the general public. As one respondent reported, data are "highly mystified in this country, often considered as something which is there only for the researchers and highly mathematical people. It has become something you have to evade and run away from." However, once data are demystified, the general public can be empowered to seek information and use it to hold policymakers accountable. This creates an internal pressure for data that could augment the growing external demand and reinforce the move toward democratization and decentralization.

\section{Lack of access to existing data}

Even when people are aware that data exist, they do not always have access to them. A number of factors seem to have discouraged the release of routine reports and the distribution of micro-data: fears of misuse, a culture of secrecy, a need to maintain confidentiality, and a lack of advance planning for sharing. These factors curb the demand for data.

Interviewees cited fear of misuse of data as a constraint to sharing. One researcher voiced this concern by saying that if someone asked for his data, "I would definitely hesitate. The reason is that I think that the data could be wrongly used." This view was taken to an extreme through the words of another respondent: "It is like a child asking you for car keys. You can't give them because you don't know what the kid is going to do with the engine." Concurrent with, and perhaps underlying, this fear of misuse is a fear of being judged; another interviewee stated: "We seem to fear criticism, we fear to be scrutinized because it may work against us." In addition, lack of confidence in the data, and in their reliability in particular, raises anxieties about sharing. Interestingly, although our interviewees repeatedly cited fears of misuse, they provided no concrete examples of such misuse.

Fears of data misuse and lack of confidence in data often co-exist in a culture of secrecy in which data are kept behind closed doors. In two of the four countries we studied, respondents referred to an institutional culture of secrecy that impedes sharing

Ghana Info strives to provide one-stop shopping for social and economic data. Data on this website are organized by sector goal and themes, and the data are disaggregated (by gender, rurallurban, district) wherever possible. of information. Interviewees mentioned that in order to combat this secrecy, people must rely on personal contacts to access data. This was reported as a special problem for younger journalists - and no doubt researchers as well-who were shut out because they lacked a network of personal contacts. Such contacts may lead to greater access for the better-connected, but reliance on personal networks does not constitute a coherent and transparent system.

Many interviewees noted that some data must be kept confidential to protect the privacy of individuals. Confidentiality is a widely shared goal and a well-understood problem with data sharing. Suppressing identifying information may be difficult to implement after the data have 
been collected, thus making the resulting data set difficult to use. Strategies for sharing data must take into account the need for confidentiality.

Another factor limiting sharing is that it is often an afterthought, and retrofitting can present many challenges. It is far easier and more cost-effective to plan data-sharing strategies in advance, so that issues of confidentiality, consent, documentation (including protocols, tools, tabulations, reports, and codebooks), and the appropriate file structure can be worked out. This is the approach used in some international surveys. Respondents in all four countries mentioned high staff turnover in statistical offices, which further underscores the need to support and document processes of data access and management from the beginning.

\section{Quality and timeliness of data}

Quality of data was reported to be uneven within and across the surveys conducted in the four countries. Respondents were particularly dismayed by inconsistencies in age breakdowns and by inexplicable gaps between waves of data collection. Clearly, the many ongoing efforts at improving data quality and at standardization of data elements and terminology are badly needed: "Ethiopia's National Strategy for Statistical Development offers a valuable opportunity to strengthen data use, as it addresses many of the issues that limit use-quality, accessibility, relevance, etc." One common observation is that when data collectors themselves are unaware of the importance of sound data, both timeliness and quality may be compromised.

Delays in the collection and release of data can effectively eliminate the demand for data from policymakers and the media. Timeliness is essential to usefulness. As one government official said: "Timeliness is a serious problem. The interval between data collection and reporting often is longer in nationwide surveys and censuses. By the time data are collected, analyzed, and the findings released, the context may have changed and the data become less useful." Policymakers who are under pressure to produce a development plan may need an analysis more quickly than analysts can produce it. Timeliness is a particular concern for members of the media when they work on strict deadlines. A breaking story might be significantly enhanced by data, but the opportunity for doing so is lost when data producers are reluctant (or unable) to respond in the time available. Admittedly, the media and other data users often have unrealistic expectations about how quickly good-quality data can be produced and released. If producers of data appear nonresponsive, this may dampen policymakers' enthusiasm for supporting the infrastructure and project costs for data collection.

\section{Lack of data}

Demand for data cannot be assessed in isolation: the supply of data inevitably affects the demand for it. Aside from the census, local-level data are sparse in the statistical systems of these four countries. While the DHS, the Multiple Indicator Cluster Survey (MICS), and similar surveys are valued, these nationally representative surveys cannot go far in addressing local data needs. To the extent that policymakers depend on such large-scale surveys, they may underinvest in (or fail to appreciate) the many data needs at local levels to monitor trends, provide the basis for supporting budget requests, or evaluate progress. When the data do not exist, or where 
the resources are not available to mine them, the fallback position may be simply not to use data at all in planning. If central governments lack data useful at the local level, they may mechanically adjust the budgets of lower-level governments without taking into account the real needs at these levels. The benefits of decentralization and results-based budgeting are thereby lost. Without access to disaggregated data, the larger goals of democratization and decentralization are unlikely to be met.

\section{Resources}

It is clearly understood that the supply of data is inevitably affected by the available resources, both human and technical. Our interviewees repeatedly mentioned insufficient skills among individuals who are involved in data collection and management. In one country, it was reported that only two or three competent statisticians are produced annually, and perhaps only one or two demographers are trained. Furthermore, respondents in all four countries reported that not all trained individuals remain in government, and some even leave the country.

In addition to these human resource constraints, technical difficulties arise. Surveys are often left in paper form and are therefore susceptible to damage. Even when technology exists, inadequate resources present a further challenge to the supply of data. Erratic electrical power supplies, poor equipment, and unreliable bandwidth impede both access to and supply of data. We heard accounts of data that are collected but not available for those who could make use of them, of data that are allowed to deteriorate because of poor storage and maintenance, and of data that are simply wrong or useless. In resource-constrained

In Senegal, the Statistics and Demographics

Department within the

National Institute for

Applied Economics (DSD-

ENEA) now falls under the purview of the national statistical agency. $D S D$ ENEA is in the process of becoming the École Nationale Supérieure de Statistique et d'Économie Appliqueé (ENSEA), whose aim is to train statisticians to work in francophone countries. settings, it can be difficult to make a case for further data collection when previously collected data lie unused. A development partner stated the need to prioritize data requirements and to "collect data on a few critical indicators." The importance of resources should not be understated. At the same time, one researcher noted that adequate resources tend to follow increased awareness: "Resources are not the first problem. First you have to get people interested in data; if they are convinced, the means to support the research can be found."

\section{Missing links between producers and users}

Often, data are available but the format in which they are provided prohibits full and easy use. Micro-data are rarely useful to policymakers and the general public; lacking training in analysis, policymakers need to have the data translated for them into more comprehensible formats, such as tables or maps. One government official extolled the virtues of maps: "When it comes to our politicians, they need to look at this picture [map] on the back of the wall and see 
that in [one district] there are about 20 primary schools constructed by government and in [another district] there is only one, so there is justification to allocate to the district which has been marginalized for all those years."

For the media, the format in which information is made available is particularly critical. Journalists much prefer lay language to statistical language. As one media interviewee stated, "Few people, even journalists, understand what the poverty index means, so instead of saying the poverty index is this or that, they could talk in terms of, for example, one in three Senegalese is poor, or half of the population cannot afford to eat."

There was strong support for data provided in user-friendly formats so that multiple audiences can make effective use of the information. Few members of constituencies (media, civil society, parliamentarians) could be expected to seek out and manipulate micro-data. Nevertheless, micro-data need to be made available to enable these different groups to construct and present their analysis in meaningful ways.

If policymakers are to use data successfully, a trusted intermediary organization with strong analytical skills must facilitate the process. We observed different ways to mediate the links between data analysts and users: a governmental entity (not unlike the Congressional Research Service in the United States) could serve as a resource; or, as some respondents mentioned, policymakers could add research assistants to their staffs to ensure that their needs are met. It would be worthwhile to investigate whether such trusted intermediaries would produce only the data (or evidence or information) that the policymaker requests, or whether they would be empowered to help frame questions. In Uganda, parliamentarians are provided with research assistants and library and internet services, but use of these resources remains low. Thus, while tools to empower policymakers may be necessary, they may not be sufficient in themselves to accelerate demand for data.

Likewise, respondents repeatedly noted that there was little dialogue between the producers and consumers that could build a constructive understanding of each other's needs, strengths, values, and limitations. Strengthening this link would allow civil society to use data for advocacy purposes and to hold governments accountable. As one NGO interviewee said, "Quality information used by the media is the most effective advocacy tool." A member of the media noted that budget data are important to them: "Journalists and the public have no idea how much money is spent on poverty reduction; to appreciate the efforts being made to reduce poverty, it is important to know how much is being spent."

\section{IMPlications OF THE CASE STUdies}

The case studies of four sub-Saharan African countries highlighted two types of issues: those that require long-term resources and those that require leadership and sometimes political will.

\section{Doing more with what we have}

Proactive dissemination of existing data. In many settings, data are not being fully utilized - indeed, their existence may not be widely known. A targeted initiative (focused on the 
most valuable data sets) could get the data into the hands of users in forms they can understand and apply, help to bring data to policymakers, and enhance awareness of the value and existence of data.

"One-stop shopping" was a frequent request. Even a modest approach that identifies what is available, what it contains, and how to gain access to it would be widely welcomed and is probably a key step in changing the culture of data use and sharing. However, the diversity of potential users makes it unlikely that any single site (unless designed to have multiple "faces") could reach all users. The analyst who needs complex data sets with weighted samples is so far removed from the reporter who wants to track politicians' promises for budget allocation that the ideal of a "single site for all data" may be unattainable.

User-friendly formats. Demand may well come from those who are not sophisticated analysts and users. Therefore, initiatives to increase their appreciation and use of data will require ways to present data in summary form, user-friendly formats, and, often, on paper. A request for "user-friendly" data is in essence a request for "middleware"-organizations and competent analysts who translate large, complex data sets into relatively simple displays without distortions, biases, and errors. One specific aspect of accessibility that requires attention is the presentation of data through maps. Maps can make data accessible to members of the media, who often have limited statistical skills, and to policymakers, who typically have a good grasp of the national or local geography and want to see how their constituencies are served relative to others.

Disaggregate data to the most useful levels. Data sets presented at a high level of aggregation frustrate many potential users. Perhaps the most urgently needed form of disaggregation is geographic: disaggregated data are useful for policymakers at local levels, enabling them to access the evidence base. Presentation in the form of maps requires that the country create digitized files of the boundaries of political and administrative units at a high degree of disaggregation. Many countries already have such files or are in the process of creating them. Once this initial investment has been made, the boundary files can permit the display of many types of data. Disaggregation by gender or other characteristics is also important and may make the difference as to whether a potential user finds data relevant or not. In some cases, disaggregation can be done with existing data, even if it is not done routinely. In other cases, data need to be collected in different ways, sometimes with very simple additions or changes in data collection procedures. In other words, the cost of providing data in more useful ways may be minimal.

Facilitate communication between producers and consumers. Data producers, analysts, and consumers are not necessarily in close contact with one another. There is a need for ways to bring them together to create a greater sense of the value of collecting and disseminating data; and in this way a realistic appreciation of the challenges in getting data could develop. Such communication might help to foster a community of data users, regardless of where they fall on the continuum of production/use of data.

Sharing data as a retrospective activity. Many data collection activities do not begin with the idea that the data will be shared, or shared beyond a narrow range of users; yet the data may be of more general value than the collectors realize. To be sure, retrofitting data so that they are 
"accessible" can be costly and therefore should not be undertaken without an assessment of the benefits relative to the costs. However, data sets that are either ready for sharing or easy to make ready could be shared, and their emergence might signal the beginning of a new approach to data access and sharing.

\section{How can we shift the paradigm?}

Strengthening intermediaries. Many intermediaries stand between policymakers and data collectors. Some are close at hand and may possess considerable technical skills, for example the central statistics office and statistical units of some ministries. Others are further removed from policymakers, but nevertheless quite salient, such as civil society groups. The academic research community may also serve the intermediary role when they are called upon—or take it upon themselves - to interpret the evidence base for policymakers. And the media can play a role in explaining policies, exploring their impact, and enhancing accountability. Repeatedly in the case studies, such intermediaries surfaced as an entry point to support the greater use of data and the evidence base in the policymaking process. However, intermediary groups face many problems in gaining access to data and applying appropriate skills to interpret them. Strengthening the skills of the intermediaries could be one strategy for developing stronger indigenous demand for data.

Would an "innovation fund" help? The case studies clearly demonstrated a high degree of interest in data and awareness of the challenges entailed in having data widely shared and used. Would an innovation fund help develop appropriate tools? While competition for funds can be cumbersome to manage, the benefits of such innovative, grass-roots approaches can be manifold. Internal demand can engender a sense of "local ownership" in the process of prioritizing data, creating data, and using data. Are we missing ways to advance innovative approaches?

Can the talent pool be altered? There is always a need for trained personnel, and a myriad of interventions are designed to provide such training. Ideally these interventions would focus not only on the usual cadre of "data professionals" but also on individuals who work in a hands-on way with data-for example, in data collection and entry. It is unlikely that these positions would be filled completely within-country in the short term. As students look for meaningful activities, could a "Demographic Peace Corps" provide a stronger bridge between the countries with greater pools of skilled workers and countries in sub-Saharan Africa that lack access to such expertise?

How could we use new technologies? The demographic sciences have an opportunity to make innovative use of new technologies such as the audio web. Such an approach recognizes that not all cultures are predominantly reading cultures. How can we bridge the often significant technological divide in linking policymakers, civil society, media, and other potential "consumers" to new data? The value of data in advancing democratic processes is considerable, but for now the gulf between traditional ways of presenting data and the new users of data remains wide. 


\section{Non-technical interventions}

Establish access to data as a right. Policy-level interventions can set the stage for expectations about data access and use. This is clearly the case in Ghana, where a Right to Information bill is pending, modeled on similar provisions in India. Of course, as important as this step is in setting high expectations for data accessibility, legislation still needs to be accompanied by many other steps (as noted above) to make data functionally available.

Leadership among the donor community is needed. Donors increasingly require that data be made available from projects they fund. However, few donors match this requirement with specific support for the task. Are there ways to ensure effective sharing that focus grantees' attention on the use of available data? Requirements should be specific so as not to impede the collection of new data when needed.

Will decentralization eventually force the data enterprise to change? Many countries are decentralizing their policy and programmatic activities in ways that place greater pressure on local areas (and policymakers) to quantify their needs, their activities, and their impact. The problems that plague national-level data are greater at the sub-national levels, and the skills needed to make use of such data are generally scarcer. And, in some cases it is not clear that the devolution of responsibility is accompanied by the authority to obtain the necessary data and the support needed to sustain an evidence-based approach to policymaking and evaluation. Our observations about ways to create greater access apply with greater emphasis at the local level.

\section{CONCLUSION}

The case studies of four sub-Saharan African countries have demonstrated the considerable interest in and the growing demand for data, although in these and other countries the link from data to policy can be far from clear. Access to data is limited by characteristics of the data, lack of expertise among the users, and inadequate systems for making data available. The challenge of greater access to and demand for data is a social-cultural problem with many technical aspects. It is always difficult to determine where to begin on such complicated issues, but a number of activities are underway to address the technical constraints. It is more difficult to conceptualize and operationalize activities to inculcate a culture of sharing or a culture of data use. There are, however, promising starting points. For example, donors can require that data collected with their support be made available to others in forms that they can use to conduct further analyses. A number of donors already make this a requirement, but real advances will come only when requirements are accompanied by strategies to financially support such activities, share technical advice, and publicize the resulting data sets. Providing research support that is restricted to those who make use of already-collected data would shift the incentives from collecting new data to maximizing the use of existing data. There are legitimate disincentives to sharing that must be overcome.

How can decisionmakers be encouraged to make more use of data in the policymaking process? Clearly data must be made accessible in forms that are user-friendly to a wide variety of policymakers who have no technical skills. An interface between the data producers, analysts, and policymakers can be created in different ways, each with its particular technical, human, and 
resource considerations. Also, there are many potential voices in the process of policy development. Entities such as NGOs and civil society, the media, and a wide array of public and private interests could all be better informed by data that help to define problems, structure interventions, and assess impact. Just as with policymakers themselves, these entities need access to data in user-friendly formats.

Is there one ideal way to share data that will lead to increased use and eventually greater demand for data? This answer, in short, is no. While some would advocate that all data be made available through the internet, others will continue to face bandwidth and power problems. However, the more data that are made widely available in a variety of formats, the greater the number of intermediaries who can be engaged. It is unlikely that any one entity can provide data in ways to meet such a wide range of needs. Our interviewees repeatedly reinforced the impression that potential users simply do not know what data are available. Yet a number of useful data sources already exist. It is equally unlikely that there is any one way to convey the message about what is available (along with sufficient technical support) and how to use it. However, there is considerable value in looking at creative ways to build an understanding of what can be done with what exists. This would also likely increase the constituencies who would lobby for more and better data and greater access to it. A virtuous circle has to begin somewhere.

The democratic process assumes that citizens are involved in the activities of governance. Evidence about the population, the economy, health, education, welfare, and other development measures is fundamental to democratic engagement. Evidence about how the government is allocating resources and the effects of those allocations is fundamental for voters to make informed decisions. Policymakers can-even with the best of intentions-make unwise allocations if they are unable to access accurate, timely information. In one country we studied, a member of an NGO noted that there was "demand for data by the various ministries and development partners in the country but that demand may not reflect the reality on the ground because there is no participatory process in deciding the data/indicators to monitor programs."

For some, this issue is catalyzed by legislation (in Ghana, the Right to Information bill) or the view that access to data is a right that all citizens should enjoy. In other cases the plea was for the democratization of data. Data are key to accountability. In these cases there is a plea for an open marketplace of data in support of an open market place of ideas.

There are many technical issues, and most are being addressed in one form or another. But the overarching challenge is not technical; it is the need for a cultural acceptance of the requirement for evidence-based policymaking. The technical problems that underlie data sharing and access to and use of data can be solved, but not without the political will to do so. Political will is more likely to be forged through broad engagement with these issues, rather than through the efforts of technical data producers/users alone. The provision of data whets the appetite for more information; and the use of data by policymakers rewards the efforts of those who collect and supply data and probably supports them in other critical ways such as financial backing. When the lack of apparently useful data reduces policymakers' interest in data, and the lack of interest in data marginalizes the data producers, the stage is set for the continuation of the status quo. Can bold steps to make data available to wider audiences, enhance incentives to make full use of existing data, and increase the exchange between producers and users lead to an open 
marketplace of data? Do efforts to improve one part of the process help to pull along other parts of the process such as training, resources, and equipment? The four case study countries offered many insights about interest in data and obstacles in the path to getting and using data.

\section{NoTES}

1 The Demographic Data for Development project is part of a broader initiative of the William and Flora Hewlett Foundation: Improving Access and Use of Demographic and Related Data for Development. This initiative is an exploratory grantmaking effort to make demographic and economic data more accessible to researchers, and to ensure that the information is effectively used by policymakers throughout sub-Saharan Africa and globally. The long-term goal is to provide policymakers with essential analyses and expand knowledge about the lives of poor people in sub-Saharan Africa, especially the urban poor, and the best ways to improve their well-being. To do this, Hewlett is engaged with ongoing efforts to expand data access and use, examine the role of promising technologies, and capitalize on the insights from country-level case studies to strengthen both national and regional data efforts.

2 The Partnership in Statistics for Development in the 21st Century (PARIS21) was founded in 1999 by the United Nations, the European Commission, the Organisation for Economic Co-operation and Development, the International Monetary Fund, and the World Bank. PARIS21 seeks to develop a culture of evidence-based policymaking and implementation to improve governance and government effectiveness in reducing poverty and achieving the Millennium Development Goals.

$3 \quad$ Health Metrics Network (HMN) is a global partnership focused on strengthening health information systems. Based at the World Health Organization, HMN and its network of partners engage in a variety of work highlighting how accurate, reliable information leads to effective decisionmaking resulting in better health outcomes. 


\section{Poverty, Gender, and Youth Working Papers}

If still in print, single copies of up to three working papers from 1989 through 2003 are available free of charge.

Beginning with the 2004 issues, working papers are no longer available in print format. Instead they are distributed electronically. As each new paper is completed, subscribers are notified by e-mail and a link to the paper is provided.

To subscribe to the Poverty, Gender, and Youth working paper e-mail notification list, or to obtain back issues from 1989 to 2003, please send your request to pgywp@ popcouncil.org.

PDFs of recent issues are available at www.popcouncil.org/publications/wp/index.html

2009

13 Wendy Baldwin and Judith Diers, "Demographic data for development in sub-Saharan Africa."

2008

12 Sajeda Amin and Lopita Huq, "Marriage considerations in sending girls to school in Bangladesh: Some qualitative evidence."

11 S. Chandrasekhar and Abhiroop Mukhopadhyay, "Multiple dimensions of urban well-being: Evidence from India"

10 Sajeda Amin and Luciana Suran, "Terms of marriage and time-use patterns of young wives: Evidence from rural Bangladesh.”

9 John Bongaarts, Thomas Buettner, Gerhard Heilig, and François Pelletier, "Has the HIV epidemic peaked?"

8 Barbara S. Mensch, Paul C. Hewett, Richard Gregory, and Stephane Helleringer, "Sexual behavior and STI/HIV status among adolescents in rural Malawi: An evaluation of the effect of interview mode on reporting."
7 John Bongaarts, "Fertility transitions in developing countries: Progress or stagnation?"

6 Cynthia B. Lloyd, "The role of schools in promoting sexual and reproductive health among adolescents in developing countries."

5 Ann Biddlecom, Richard Gregory, Cynthia B. Lloyd, and Barbara S. Mensch, "Premarital sex and schooling transitions in four sub-Saharan African countries."

4 Sajeda Amin, John B. Casterline, and Laura Spess, "Poverty and fertility: Evidence and agenda."

3 Bussarawan Teerawichitchainan and James F. Phillips, "Ethnic differentials in parental health seeking for childhood illness in Vietnam."

2 Zachary Zimmer, Kim Korinek, John Knodel, and Napaporn Chayovan, "Support by migrants to their elderly parents in rural Cambodia and Thailand: A comparative study."

1 Sharon Ghuman and Cynthia B. Lloyd, "Teacher absence as a factor in gender inequalities in access to primary schooling in rural Pakistan." 